\title{
Complex genetic alterations contribute to rapid disease progression in an ALK rearrangement lung adenocarcinoma patient: a case report
}

\author{
Xiang Long ${ }^{1}$, Hao $\mathrm{Wu}^{2}$, Chenglin Yang ${ }^{3}$, Fang $\mathrm{Li}^{4}$, Min Zhang ${ }^{4}$, Xuan $\mathrm{Wu}^{5,6}$ \\ ${ }^{1}$ Department of Respiratory and Critical Care Medicine, Peking University Shenzhen Hospital, Shenzhen, China; ${ }^{2}$ Department of thoracic surgery, \\ Shenzhen Second People's Hospital, The First Affiliated 7 Hospital of Shenzhen University, Shenzhen, China; ${ }^{3}$ National Cancer Center/National \\ Clinical Research Center for Cancer/Cancer Hospital \& Shenzhen Hospital, Chinese Academy of Medical Sciences and Peking Union Medical \\ College, Shenzhen, China; ${ }^{4}$ Department of Medical Center, Geneplus-Beijing, Beijing, China; ${ }^{5}$ Department of Medical Oncology, Peking University \\ Shenzhen Hospital, Shenzhen, China; ${ }^{6}$ Cancer Institute of Shenzhen-PKU-HKUST Medical Center, Shenzhen, China \\ Correspondence to: Xuan Wu. Department of Medical Oncology, Peking University Shenzhen Hospital, Shenzhen 518036, Guangdong Province, \\ China. Email: whohol@163.com.
}

\begin{abstract}
Anaplastic lymphoma kinase tyrosine kinase inhibitors (ALK-TKIs) have been found to significantly improve the quality of life and survival in $A L K$-positive non-small cell lung cancer (NSCLC) patients. However, the duration of responses is limited by drug resistance. Genetic heterogeneity of $A L K-$ positive tumors could potentially explain the differences in individual patient outcomes. We performed nextgeneration sequencing (NGS) on plasma samples, pleural effusion samples, and tissue re-biopsy obtained at various treatment milestones from an $A L K$ rearrangement lung adenocarcinoma patient undergoing targeted therapy. The liver metastases of the EML4-ALK NSCLC patient presented rapid progression after 3.5 months of alectinib, while the other lesions showed good partial response. Targeted NGS identified the newly emerged MET amplification except for EML4-ALK in plasma ctDNA and liver lesions. Subsequently, a clinical benefit was achieved one month after the commencement of crizotinib, a dual ALK and MET inhibitor; however, the patient experienced disease progression another month later. Several rounds of ALKTKI combination therapy were tried but failed. Concurrent genetic alterations, including loss-of-function mutations in $F B X W 7$ and $M L L 3$, may mainly contribute to poor prognosis in the patient. It highlighted the molecular profiling by using NGS can be useful in identifying the heterogeneity across lesions and the resistance mechanism of targeted treatments.
\end{abstract}

Keywords: Anaplastic lymphoma kinase (ALK); MET amplification; resistance mechanism; next-generation sequencing (NGS); case report

Submitted Dec 20, 2020. Accepted for publication Mar 05, 2021.

doi: $10.21037 /$ tcr-20-3473

View this article at: https://dx.doi.org/10.21037/tcr-20-3473

\section{Introduction}

Anaplastic lymphoma kinase $(A L K)$ gene rearrangements have been reported in approximately $5 \%$ of non-small cell lung cancers (NSCLCs) and function as oncogenic driver event $(1,2)$. The second-generation ALK inhibitor alectinib demonstrated superior efficacy and lower toxicity compared to the first-generation ALK inhibitor crizotinib in advanced $A L K$-rearranged NSCLCs (3), establishing alectinib as the new standard first-line therapy. The sequential therapy of ALK-tyrosine kinase inhibitors (TKIs) allows long survivals up to more than 7 years $(4,5)$. Despite responding to ALK-TKIs initially, rapid progression may have occurred, thereby limiting the prolonged effectiveness of ALKTKIs. Genetic heterogeneity of $A L K$-positive tumors could potentially explain the differences in individual patient outcomes (6). Here, we present a case of an $A L K$-rearranged 
NSCLC patient who had initially benefited from alectinib and crizotinib before the disease rapidly progressed. We identified some concurrent mutations by panel sequencing which possibly conferred resistance to ALK-TKIs. We present the following case in accordance with the CARE reporting checklist (available at https://dx.doi.org/10.21037/ tcr-20-3473).

\section{Case presentation}

A 39-year-old man, a former smoker (10 cigarettes/ day), with no significant past medical and family history, presented to our hospital for chest and back pain that had persisted for 2 weeks. A chest computed tomography (CT) scan revealed a lesion in the upper lobe of his right lung. Pathologic analysis of bronchoscopy biopsy confirmed lung adenocarcinoma in April 2019. The clinical stage was IV due to extensive metastases (bilateral lung, liver, retroperitoneal lymph nodes, etc.). The tumor tissue was sent for genomic testing using targeted DNA sequencing (1,021 cancer-related genes) as well as pleural effusion circulating tumor DNA (ctDNA) (Supplementary Method), as described previously $(7,8)$. Echinoderm microtubuleassociated protein-like 4 (EML4)-ALK (E13:A20) fusion was identified with a mutant allele frequency (AF) of $15.5 \%$ in tissue and $31.2 \%$ in ctDNA (Table 1). The patient received alectinib (600 mg twice a day) since May 2019 and achieved partial response to liver metastasis [Response Evaluation Criteria in Solid Tumors (RECIST) v1.1] after 1 month (Figure 1). However, the CT scan showed a dramatic progression of liver metastasis after 3.5 months of treatment, while the other lesions showed good partial response. Then the patient was treated with radiofrequency ablation of liver tumors, but the liver lesions continued to grow rapidly. Meanwhile, the next-generation sequencing detected both EML4-ALK rearrangement and MET amplification in plasma ctDNA and liver lesions (Table 1). Crizotinib (250 mg twice a day), a dual inhibitor of ALK and MET, was then administered in September 2019. Symptoms such as chest and back pain significantly improved within the first month, and the diameter of the liver metastases decreased from 66 to $50 \mathrm{~mm}$. But unfortunately, the patient developed multiple low-density nodules in the liver in November 2019. To explore new therapeutic strategies, we used ctDNA analysis to track the evolution of resistance during treatment. The result revealed retained EML4-ALK fusion $(\mathrm{AF}=14.5 \%)$ without the amplification of MET (Table 1). Since the patient experienced further disease progression with new bone lesions, pemetrexed, cisplatin, and bevacizumab were given as third-line treatment. After two cycles of chemotherapy, liver metastases, especially those in the left lobe, progressed again, so the patient switched to a combination treatment of alectinib and cabozantinib (60 mg twice a day), a multikinase inhibitor with activity against MET, in March 2020. He achieved stable disease (SD) after two months, and cabozantinib discontinued due to grade 3 hand-foot syndrome (HFS). At that time, a second biopsy specimen showed low PD-L1 expression with a tumor proportion score (TPS) of $1-2 \%$ by immunohistochemistry (Dako 22C3). The patient had shown increased ctDNA gene mutation frequencies while the amplification of MET was still not detected (Table 1). Based on the results from the phase III ALTER-0303 trial (Clinical Trial Registry ID: NCT 02388919) of anlotinib in China, anlotinib (12 mg) and alectinib were administered, but the patient's condition continued to deteriorate. Repeated analyses indicated the presence of an inactivating mutation in FBXW7 (p.M268Dfs*18) during the disease (Table 1) which may be sensitive to mTOR inhibitor (9). After a discussion with the patient and approval from his insurer, he was treated with lorlatinib $(100 \mathrm{mg})$ and everolimus (10 mg) in May 2020. After 20 days of treatment, the diameter of the liver metastases decreased from 225 to $167 \mathrm{~mm}$. However, the disease had substantially progressed and the patient died on June 15, 2020. Figure 1 illustrated the flow of treatments and image evaluation.

All procedures performed in studies involving human participants were in accordance with the ethical standards of the institutional and/or national research committee(s) and with the Helsinki Declaration (as revised in 2013). Written informed consent was obtained from the patient.

\section{Discussion}

Though the number of $A L K$-rearranged lung cancer patients is relatively small, multiple ALK-TKIs have a relatively longer progression-free survival (PFS) period and have been approved for clinical use. Alectinib, a second-generation ALK-TKI, improves prognosis of treatment-naive ALKpositive NSCLCs, with an objective response rate of $82.9 \%$ and median PFS of 34.8 months $(3,10)$. However, resistance to TKIs is inevitable and the mechanism of acquired resistance to alectinib in patients with $A L K$ rearrangement has not yet been completely identified. In our case, the patient showed a mixed response to liver metastasis and primary lung tumors after initial alectinib treatment 

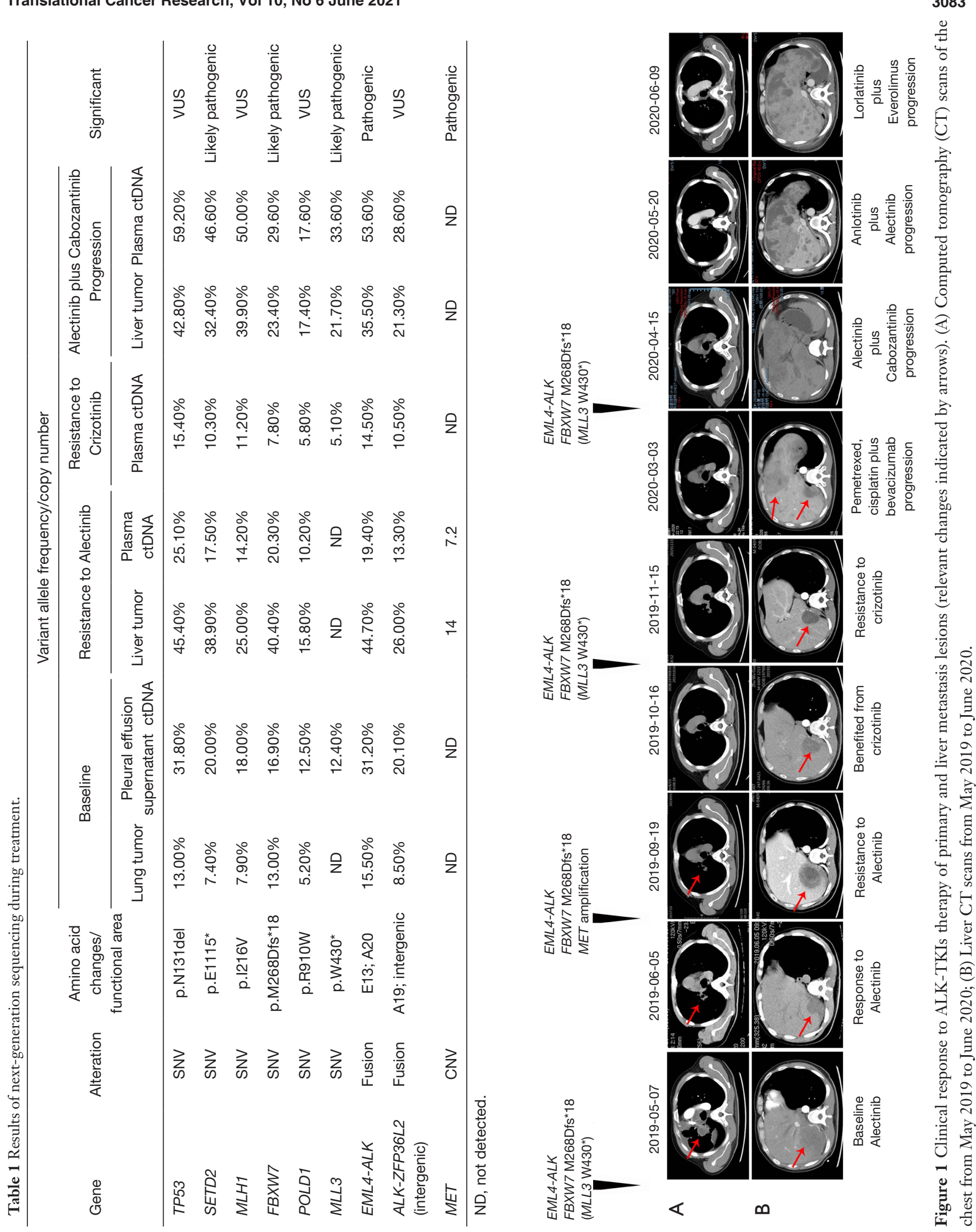
(Figure 1). This phenomenon may attribute to intertumoral genetic heterogeneity. To clarify the resistance mechanism, a panel sequencing was performed in the liver metastasis. In addition to the EML4-ALK fusion previously discovered in the primary lesion, $M E T$ amplification was detected. Previous studies have found evidence that cMET activation through $M E T$ gene amplification can potentially confer resistance to alectinib but not to crizotinib $(11,12)$. Our case showed a clinical benefit of crizotinib despite of drug resistance that occurred rapidly after. A negative result for the amplification of $M E T$ was found in the cfDNA at that time, which may be due to non-shedding of the amplified $M E T$, real elimination of the amplified clone, or too little ctDNA in the plasma sample. Considering the absence of the amplification of $M E T$ was confirmed in rebiopsies from hepatic biopsies after the progression of alectinib and cabozantinib, we cannot exclude that crizotinib caused or contributed to the disappearance of the MET amplification tumor clone, as we did observe a response of some hepatic lesions to this drug.

Except for $M E T$ amplication, some key variants were list in table1, especially those present from baseline throughout the time course. Their pathogenicity and association with ALK-TKIs resistance were assessed using the public databases and published literature, such as ClinVar, Catalogue of Somatic Mutations in Cancer (COSMIC), and PubMed. Previous studies suggested a potential role of TP53 mutations in poor therapeutic response and outcome in ALK/TP53 co-mutated patients $(6,13,14)$. The deletion of $F B X W 7$ in NSCLC was also found associated with poor overall survival $(15,16)$. FBXW7 is a member of the F-box protein family, which controls proteasome-mediated degradation of oncoproteins such as rapamycin (mTOR), c-Myc, cyclin E, Mcl-1, Jun, and Notch 1 (17). In vitro studies showed that the loss of FBXW7 leads to resistance to gefitinib and crizotinib $(16,18)$. Villaruz et al. reported a case that harbored an $F B X W 7$ mutation without EGFRmutant or ALK rearrangement responded to the mTOR inhibitor temsirolimus (9). In our case, a combination of ALK-TKI and mTOR inhibitor did not seem to overcome ALK-TKI resistance, potentially due to posterior line of therapy or different choice of mTOR inhibitor. Moreover, Ye et al. reported a PI3K/Akt- and MEK/Erk-independent resistance mechanism by which loss of FBXW7 leads to targeted therapy resistance via stabilization of anti-apoptotic protein Mcl-1 (18), suggesting that FBXW7-mediated activation of multiple signaling pathways might contribute to ALK-TKIs resistance. Notably, FBXW7 inactivation is known to partly induce TKI-resistance by promoting epithelial-mesenchymal transition (EMT) (16). EMT has recently been implicated in resistance to lorlatinib in patient-derived cell lines (19) and to alectinib and lorlatinib in a patient (20). Interestingly, we identified a concomitant nonsense mutation in $M L L 3$ at relapse on Crizotinib. Some studies demonstrated the function of mutant MLL3 in facilitating tumor EMT (21) and involvement in lung cancer development and survival (22). Together, these observations indicate that EMT induced by the deletion of FBXW7 and MLL3 may represent the main mechanism of resistance to ALK-TKIs. There are not enough samples to confirm the presence of EMT by using markers such a positive immunostaining for vimentin and loss of E-cadherin expression. But we found that the third tumor rebiopsy taken from hepatic metastases had almost completely lost the expression of the adenocarcinoma-marker CK7 and TTF1. It is worth-noting that mutations crossing take place between $M E T$ and $M L L 3$, revealing the resistance heterogeneity and selection of tumor evolution.

In addition to the above speculation, non-reciprocal $A L K$ translocation with the retaining of the 5' region of the ALK gene was also observed in the patient samples (Table 1), which was reported as a poor predictive marker in first-line crizotinib-treated $A L K$-rearranged NSCLCs (23). In that study, three patients who harbored non-reciprocal/ reciprocal $A L K$ translocation also did not benefit from alectinib therapy after the failure of crizotinib. However, it is still poorly understood how 5'-ALK DNA could contribute to poor prognosis of patients.

In conclusion, we report the case of an EML4-ALK fusion-positive NSCLC patient, who progressed rapidly during the different lines of ALK-TKIs therapy in a year. Genetics variations concurrent with $E M L 4-A L K$ from tissues and ctDNA during disease may be accounted for the treatment response and prognosis of the patient. Molecular profiling by using NGS can be useful for monitoring tumor heterogeneity and clonal evolution during ALK-TKIs treatment in NSCLCs. Treatment strategises for these patients need further research.

\section{Acknowledgments}

We thank Dr. Hanxin Yao for proof reading the manuscript as a native English-speaking expert.

Funding: This work was supported by the Natural Science 
Foundation of Guangdong Grants (2016A030310242).

\section{Footnote}

Reporting Checklist: The authors have completed the CARE reporting checklist. Available at https://dx.doi. org/10.21037/tcr-20-3473

Peer Review File: Available at https://dx.doi.org/10.21037/ tcr-20-3473

Conflicts of Interest: All authors have completed the ICMJE uniform disclosure form (available at https://dx.doi. org/10.21037/tcr-20-3473). The authors have no conflicts of interest to declare.

Ethical Statement: The authors are accountable for all aspects of the work in ensuring that questions related to the accuracy or integrity of any part of the work are appropriately investigated and resolved. All procedures performed in studies involving human participants were in accordance with the ethical standards of the institutional and/or national research committee(s) and with the Helsinki Declaration (as revised in 2013). Written informed consent was obtained from the patient.

Open Access Statement: This is an Open Access article distributed in accordance with the Creative Commons Attribution-NonCommercial-NoDerivs 4.0 International License (CC BY-NC-ND 4.0), which permits the noncommercial replication and distribution of the article with the strict proviso that no changes or edits are made and the original work is properly cited (including links to both the formal publication through the relevant DOI and the license). See: https://creativecommons.org/licenses/by-nc-nd/4.0/.

\section{References}

1. Soda M, Choi YL, Enomoto M, et al. Identification of the transforming EML4-ALK fusion gene in non-small-cell lung cancer. Nature 2007;448:561-6.

2. Devarakonda S, Morgensztern D, Govindan R. Genomic alterations in lung adenocarcinoma. Lancet Oncol 2015;16:e342-51.

3. Camidge DR, Dziadziuszko R, Peters S, et al. Updated Efficacy and Safety Data and Impact of the EML4-ALK Fusion Variant on the Efficacy of Alectinib in Untreated ALK-Positive Advanced Non-Small Cell Lung Cancer in the Global Phase III ALEX Study. J Thorac Oncol 2019;14:1233-43.

4. Duruisseaux M, Besse B, Cadranel J, et al. Overall survival with crizotinib and next-generation ALK inhibitors in ALK-positive non-small-cell lung cancer (IFCT-1302 CLINALK): a French nationwide cohort retrospective study. Oncotarget 2017;8:21903-17.

5. Watanabe S, Yamanaka T, Ito K, et al. OA02.06 The Sequential Therapy of Crizotinib Followed by Alectinib: Real World Data of 840 Patients with NSCLC Harboring ALK-Rearrangement (WJOG9516L). J Thorac Oncol 2019;14:S209-10.

6. Kron A, Alidousty C, Scheffler M, et al. Impact of TP53 mutation status on systemic treatment outcome in ALKrearranged non-small-cell lung cancer. Ann Oncol 2018;29:2068-75.

7. Nong J, Gong Y, Guan Y, et al. Circulating tumor DNA analysis depicts subclonal architecture and genomic evolution of small cell lung cancer. Nat Commun 2018;9:3114.

8. Lu C, Dong XR, Zhao J, et al. Association of genetic and immuno-characteristics with clinical outcomes in patients with RET-rearranged non-small cell lung cancer: a retrospective multicenter study. J Hematol Oncol 2020;13:37.

9. Villaruz LC, Socinski MA. Temsirolimus therapy in a patient with lung adenocarcinoma harboring an FBXW7 mutation. Lung Cancer 2014;83:300-1.

10. Peters $S$, Camidge D, Shaw A, et al. Alectinib versus Crizotinib in Untreated ALK-Positive Non-Small-Cell Lung Cancer. N Engl J Med 2017;377:829-38.

11. Gouji T, Takashi S, Mitsuhiro T, et al. Crizotinib can overcome acquired resistance to CH5424802: is amplification of the MET gene a key factor? J Thorac Oncol 2014;9:e27-8.

12. Isozaki $\mathrm{H}$, Ichihara $\mathrm{E}$, Takigawa $\mathrm{N}$, et al. Non-Small Cell Lung Cancer Cells Acquire Resistance to the ALK Inhibitor Alectinib by Activating Alternative Receptor Tyrosine Kinases. Cancer Res 2016;76:1506-16.

13. Song P, Zhang F, Li Y, et al. Concomitant TP53 mutations with response to crizotinib treatment in patients with ALK-rearranged non-small-cell lung cancer. Cancer Med 2019;8:1551-7.

14. Camidge D, Niu H, Kim HR, et al. Correlation of baseline molecular and clinical variables with ALK inhibitor efficacy in ALTA-1L. J Clin Oncol 2020;38:9517-.

15. Yokobori T, Yokoyama Y, Mogi A, et al. FBXW7 mediates chemotherapeutic sensitivity and prognosis in NSCLCs. 
Mol Cancer Res 2014;12:32-7.

16. Xiao Y, Yin C, Wang Y, et al. FBXW7 deletion contributes to lung tumor development and confers resistance to gefitinib therapy. Mol Oncol 2018;12:883-95.

17. Yeh CH, Bellon M, Nicot C. FBXW7: a critical tumor suppressor of human cancers. Mol Cancer 2018;17:115.

18. Ye M, Zhang Y, Zhang X, et al. Targeting FBW7 as a Strategy to Overcome Resistance to Targeted Therapy in Non-Small Cell Lung Cancer. Cancer Res 2017;77:3527-39.

19. Recondo G, Mezquita L, Facchinetti F, et al. Diverse Resistance Mechanisms to the Third-Generation ALK Inhibitor Lorlatinib in ALK-Rearranged Lung Cancer. Clin Cancer Res 2020;26:242-55.

20. Urbanska EM, Sørensen JB, Melchior LC, et al. Changing ALK-TKI-Resistance Mechanisms in Rebiopsies of

Cite this article as: Long $\mathrm{X}, \mathrm{Wu} \mathrm{H}$, Yang $\mathrm{C}$, Li F, Zhang M, $\mathrm{Wu} \mathrm{X}$. Complex genetic alterations contribute to rapid disease progression in an $A L K$ rearrangement lung adenocarcinoma patient: a case report. Transl Cancer Res 2021;10(6):3081-3086. doi: $10.21037 /$ tcr-20-3473
ALK-Rearranged NSCLC: ALK- and BRAF-Mutations Followed by Epithelial-Mesenchymal Transition. Int J Mol Sci 2020;21:2847.

21. Cho S, Yoon C, Lee J, et al. KMT2C Mutations in Diffuse-Type Gastric Adenocarcinoma Promote Epithelial-to-Mesenchymal Transition. Clin Cancer Res 2018;24:6556-69.

22. Fagan RJ, Dingwall AK. COMPASS Ascending: Emerging clues regarding the roles of MLL3/KMT2C and MLL2/ KMT2D proteins in cancer. Cancer Lett 2019;458:56-65.

23. Zhang Y, Zeng L, Zhou C, et al. Detection of Nonreciprocal/Reciprocal ALK Translocation as Poor Predictive Marker in Patients With First-Line CrizotinibTreated ALK-Rearranged NSCLC. J Thorac Oncol 2020;15:1027-36. 


\section{Supplementary Method}

\section{DNA extraction, targeted capture, and NGS}

Genomic DNA of tissue samples was extracted by using the QIAamp DNA FFPE Tissue Kit (Qiagen GmbH, Hilden, Germany), according to the manufacturer's protocol. 4-5 $\mathrm{mL}$ of plasma was used to isolate cell-free DNA (cfDNA) by using the QIAamp Circulating Nucleic Acid Kit (Qiagen, Valencia, CA). Peripheral blood lymphocytes (PBLs) were used to extract germline genomic DNA with the DNeasy Blood Kit (Qiagen, Valencia, CA). Indexed NGS libraries were constructed from sheared DNA using the DNA Library Preparation Kit for MGISeq-2000 (BGI, Shenzhen, China). All libraries were hybridized to custom-designed biotinylated oligonucleotide probes (Roche NimbleGen, Madison, WI, USA) covering 1021 genes. DNA sequencing was performed using the MGISeq-2000 Sequencing System (BGI, Shenzhen, China) per the manufacturer's guideline.

\section{Sequencing data analysis and variant interpretation}

The clean reads were mapped to the reference human genome (GRCh37) using Burrows-Wheeler Aligner (BWA), after removal of terminal adaptor sequences and low quality data. Somatic small insertions and deletions (InDels) and single nucleotide variants (SNVs) were called with MuTect and GATK. Copy-number variants $(\mathrm{CNVs})$ were identified with CONTRA 2.0.8 software, and structural variants (SV) were identified using BreakDancer. All final candidate variants were manually verified in the Integrative Genomics Viewer (IGV). 